\title{
Dynamic Path construction in Multi-Hop Wireless Networks
}

\author{
Nagineni Sharoon 1 )
}

\begin{abstract}
In the problem of routing in multi-hop wireless networks, to accomplish top of the line to-end throughput, it is vital to locate the "best" way from the source hub to the goal hub. In spite of the fact that a substantial number of steering conventions have been proposed to discover the way with least aggregate transmission check/time for conveying a solitary parcel, such transmission count/time minimizing protocols can't be ensured to accomplish most extreme end-to-end throughput. In this paper, we contend that via painstakingly considering spatial reusability of the remote correspondence media, we can colossally enhance the end-to-end throughput in multi-jump remote systems. To support our contention, we propose spatial reusability-aware single-path routing (SASR) and anypath routing (SAAR) protocols and contrast them and existing single-way steering and anypath steering conventions, individually. Our assessment comes about demonstrate that our conventions fundamentally enhance the end-to-end throughput contrasted and existing conventions. In particular, for single-way steering, the middle throughput pick up will be up to 60 percent, and for every source-goal match, the throughput pick up is as high as 5:3, for anypath directing, the greatest per-stream throughput pick up is 71.6 percent, while the median gain is up to 13.2 percent.
\end{abstract}

Keywords : routing, wireless network, protocol design, path, multi-hop, SAAR, SASR.

\section{Introduction}

Because of constrained limit of wireless communication media and lossy wireless links[1-5], it is critical to precisely choose the course that can expand the end-to-end throughput, particularly in multi-bounce remote systems. As of late, an expansive number of directing conventions (e.g., [6-10], and so forth.) have been proposed for multihop wireless networks. Notwithstanding, a central issue with existing remote directing conventions is that limiting the general number (or time) of transmissions to convey a solitary parcel from a source hub to a goal hub does not really augment the end-to-end throughput. A point by point illustration will be displayed in Section 3.2 to demonstrate this observation.

In this paper, we research two sorts of routing protocols, including single-way directing and any way directing. The assignment of a solitary way directing convention is to choose a cost

Received(April 1, 2016), Review Result(1st: April 22, 2016, 2nd: May 16, 2016), Accepted(December 10, 2015)

${ }^{1}$ (Corresponding Author) Machine Intelligence Research Labs, India

email: Satyasaikiran9@gmail.com 
limiting way, along which the parcels are conveyed from the source node to the destination node. As of late, any path steering (e.g.,[11-15]) shows up as a novel directing procedure misusing the communicate way of remote correspondence media to enhance the end-to-end throughput. It totals the force of numerous moderately feeble ways to shape a solid way, by respecting any middle of the road hub who catches the bundle to partake in parcel sending. The greater part of existing directing protocols, regardless of singlepath steering conventions or anypath steering protocols, depend on connection quality mindful directing measurements, for example, interface transmission tally based measurements (e.g., ETX [16] and EATX [17]) and interface transmission time-based measurements (e.g., ETT [18] and EATT [19]). They basically select the (any)path that limits the general transmission tallies or transmission time for delivering a packet.

\section{Proposed System}

\subsection{Related work}

In this section, we briefly review related works on metric design and protocol implementation. We also compare ourwork with those on joint routing problems, as well as other works considering reusability.

\subsection{Routing Metrics}

There are various chips away at wireless routing metrics. For single-path routing, a few connection quality mindful measurements [1][6][7][9] were proposed. RTT [20] measured the cost of single remote connection by the round outing postponement of test parcels on it; ETX [6] doled out the connection cost with its normal number of transmissions to effectively convey a bundle. In view of ETX, the creators in [9] planned ETOP metric considering connections' genuine position on the way. Moreover, joining the multi-rate capacity, ETT [7] took the normal transmission time of a connection as its cost; and EMTT [31] extended the work to multicast. Besides, gave a few standards to steering metric plan. There're additionally measurements reasonable for anypath directing [4][13]. Chachulski et al. given ETOX in [4] which considers astute gatherings at any forwarder. In the EATX metric was characterized to reflect general transmissions in any-way sending. Laufer et al. [13] received EATX as the hyperlink cost, and characterized the anypath cost made out of the hyperlink cost and the rest 
of the cost. Be that as it may, existing steering measurements have a tendency to ascertain way cost utilizing some instrument of lossless blend of connection expenses. For instance, the ETX estimation of a way is the expansion of every connection's ETX [6]. Additionally, Laufer computed the anypath cost while considering all the forwarders' expenses [13]. Additionally, the rules in [20], for example, consistency, overlooked the impact of reusability. Such lossless mechanism thus misses the opportunity of exploiting spectrum spatial reusability in wireless media.

\subsection{Routing Protocols}

The soonest single-way routing protocols [3][10][17][18] connected Dijkstra calculation for course choice. With regards to anypath routing, for instance, ExOR [2] showed up as a coordination system between forwarders; MORE [4] broke such coordination where all the forwarders worked by their workload. Plus, MORE brought system coding into anypath directing. On that premise, [13] proposed the most limited anypath first (SAF) calculation to decide the forwarders' needs, and demonstrated its optimality; [19] joined rate control and utilized a thought called credit to acknowledge stream control; CodeOR [14] empowered simultaneous transmissions of a window of fragments; SOAR [19] considered the issue of way disparity and rate confinement to effectively bolster different streams; SourceSync [20] synchronized senders to accomplish consolidated signs which brings down the bundle mistake rate. In addition, [20] built up an enhancement system to endeavor correspondence openings emerging by possibility; $\mathrm{Hu}$ et al. [8] proposed POR in view of a for each parcel input system.

Since the above directing conventions were planned in view of existing transmission cost limiting routing metrics, they can't ensure greatest end-to-end throughput when spatial reusability can't be disregarded. Furthermore, unique in relation to works, for example, [2] and [20], which ought to some degree depend on synchronization between hubs, the throughput changes of our algorithms in this work needn't bother with MAC-layer coordination.

\subsection{Existing system}

- Most of existing routing protocols, regardless of single way directing conventions or any

way steering conventions, depend on connection quality mindful steering measurements, for example, interface transmission count-based metrics and link transmission time-based metrics (e.g., ETT and EATT). They essentially select the (any) way that limits the general transmission 
Dynamic Path construction in Multi-Hop Wireless Networks

numbers or transmission time for conveying a parcel.

- Zhang et al. [16] planned joint routing and scheduling into an optimization problem and tackled the issue with a segment era technique.

- Pan et al. [16] managed the joint issue in cognitive radio networks considering the opportunity of licensed bands.

- Jones et al. [11] actualized k-tuple network coding and demonstrated throughput optimality of their strategy.

\subsection{Disadvantages of existing system}

- A fundamental problem with existing wireless routing protocols is that minimizing the overall number (or time) of transmissions to deliver a single packet from a source node to a destination node does not necessarily maximize the end-to-end throughput.

- Most of the existing routing protocols do not take spatial reusability of the wireless communication media into account.

- They need centralized control to realize MAC-layer scheduling, and to eliminate transmission contention.

\subsection{Proposed system}

In this paper, we explore two sorts of routing protocols, including single-way directing and anypath routing. The assignment of a solitary way routing protocol is to choose a cost limiting way, along which the parcels are conveyed from the source node to the destination node.

In this primer work, we contend that via deliberately considering spatial reusability of the remote correspondence media, we can immensely enhance the end-to-end throughput in Multihop wireless networks.

The algorithms proposed in this work don't require any scheduling, and the SASR algorithms can be implemented in a distributed manner.

\subsection{Advantages of proposed system}


To the best of our insight, we are the first to explicitly consider spatial reusability of the wireless communication media in routing and design practical spatial reusability-aware single-path routing (SASR) and anypath routing (SAAR) protocols.

We figure the issue of spatial reusability mindful single-path routing as a binary program and propose two correlative classifications of algorithms for way choice. While one classification (SASR-MIN and SASR-FF) tends to misuse the best execution of the ways, the other class (SASR-MAX) assesses the execution of the ways in the most pessimistic scenario.

We additionally examine the range spatial reusability in any way steering, and propose SAAR algorithm for taking part hub choice, cost count, and sending list assurance.

We have assessed SASR algorithms and SAAR calculation with various information rates. The assessment comes about demonstrate that our calculations essentially enhance the end-to-end throughput contrasted and existing ones.

Specifically, for single-way routing, a throughput pick up with a middle of more than 60 percent is accomplished on account of single-stream, and a normal pick up of more than 20 percent is accomplished with numerous streams; for anypath steering, a middle pick up of 13:2 percent and the most extreme pick up to 71:6 percent can be realized.

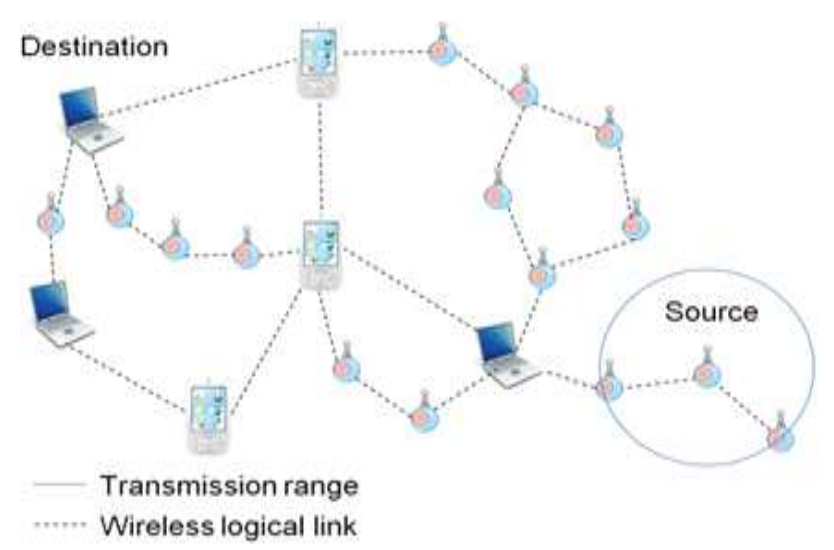

[Fig 1] System Architecture

\section{Conclusion}

In this paper, we have exhibited that we can altogether enhance the end-to-end throughput in multi-hop wireless networks by carefully considering spatial reusability of the remote 
correspondence media. We have displayed two conventions, SASR and SAAR, for spatial reusability-mindful single-way directing and anypath steering, separately. We have additionally executed our conventions and contrasted them and existing directing conventions with the information rates of 11 and $54 \mathrm{Mbps}$. Assessment comes about demonstrate that SASR and SAAR calculations can accomplish more huge end-to-end throughput increases under higher information rates. For the instance of single-stream, SASR accomplishes a throughput pick up of as high as 5:3_ under 54 Mbps, while for SAAR, the greatest pick up can achieve 71:6 percent. Besides, in multi-stream case, SASR can likewise enhance the per-stream normal throughputs by more than 20 percent. Then, the gigantic throughput increases just require adequate extra transmission overheads. The additional transmission overheads of course demand are under 10 percent in our assessment. In 80 percent cases, the general transmission numbers are expanded by close to two with SASR, while for SAAR, the greater part of the additions are beneath 1 .

With respect to the future work, one bearing is to additionally investigate chances to enhance the execution of our directing calculations by dissecting exceptional failing to meet expectations cases distinguished in the assessment. Another heading is to examine between stream spatial reusability, and to upgrade systemwide execution.

\section{References}

[1] A. Adya, P. Bahl, J. Padhye, A. Wolman, and L. Zhou, A multi-radiounification protocol for IEEE 802.11 wireless networks, Proc. 1st Int. Conf. Broadband Netw., (2004), pp.344-354.

[2] S. Biswas and R. Morris, Exor: Opportunistic multi-hop routingfor wireless networks, Proc. SIGCOMM Conf. Appl., Technol.,Archit., Protocols Comput. Commun., (2005), pp.133-144.

[3] J. Broch, D. A. Maltz, D. B. Johnson, Y.-C.Hu, and J. G. Jetcheva, A performance comparison of multi-hop wireless ad hoc networkrouting protocols, Proc. 4th Annu.ACM/IEEE Int. Conf.Mobile Comput.Netw., (1998), pp.85-97.

[4] S. Chachulski, M. Jennings, S. Katti, and D. Katabi, Trading structurefor randomness in wireless opportunistic routing, Proc. SIGCOMM Conf. Appl., Technol., Archit. Protocols Comput. Commun., (2007), pp.169-180.

[5] http://link.aps.org/doi/10.1103/PhysRevLett.90.058701, Feb 25 (2003)

[6] D. S. J. D. Couto, D. Aguayo, J. C. Bicket, and R. Morris, A highthroughputpath metric for multi-hop wireless routing, Proc. 9th Annu. Int. Conf. Mobile Comput.Netw., (2003), pp.134-146.

[7] R. Draves, J. Padhye, and B. Zill, Routing in multi-radio, multihopwireless mesh networks, Proc. 10th Annu. Int. Conf. MobileComput.Netw., (2004), pp.114-128. 
[8] W. Hu, J. Xie, and Z. Zhang, Practical opportunistic routingin high-speed multi-rate wireless mesh networks, Proc. 14th ACM Int. Symp.Mobile Ad Hoc Netw. Comput., (2013), pp.127-136.

[9] G. Jakllari, S. Eidenbenz, N. W. Hengartner, S. V. Krishnamurthy, and M. Faloutsos, Link positions matter: A noncommutativerouting metric for wireless mesh network, Proc. IEEE 27th Conf. Comput .Commun., (2008), pp.744-752.

[10] D. B. Johnson and D. A. Maltz, Dynamic source routing in adhoc wireless networks, Mobile Comput., (1996), Vol.353, pp.153-181.

[11] N. M. Jones, B. Shrader, and E. Modiano, Optimal routing andscheduling for a simple network coding scheme, Proc. INFOCOM, (2012), pp.352-360.

[12] T. S. Kim, J. C. Hou, and H. Lim, Improving spatial reusethrough tuning transmit power, carrier sense threshold, and datarate in multihop wireless networks, Proc. 12th Annu. Int. Conf. Mobile Comput. Netw., (2006), pp.366-377.

[13] R. P. Laufer, H. Dubois-Ferriere, and L. Kleinrock, Multirate Anypath Routing in Wireless Mesh Networks, Proc. INFOCOM, (2009), pp.37-45.

[14] Y. Lin, B. Li, and B. Liang, Codeor: Opportunistic routing inwireless mesh networks with segmented network coding, Proc. IEEE Int. Conf. Netw. Protocols, (2008), pp.13-22.

[15] J. Padhye, S. Agarwal, V. N. Padmanabhan, L. Qiu, A. Rao, and B. Zill, Estimation of link interference in static multi-hop wireless networks, Proc. Internet Meas. Conf., (2005), p.28.

[16] M. Pan, C. Zhang, P. Li, and Y. Fang, Joint routing and link scheduling for cognitive radio networks under uncertain spectrumsupply, Proc. INFOCOM, (2011), pp.2237-2245.

[17] C. E. Perkins and E. M. Belding-Royer, Ad-hoc on-demand distancevector routing, Proc. 2nd IEEE Workshop Mobile Comput. Syst., (1999), pp.90-100.

[18] C. E. Perkins and P. Bhagwat, Highly dynamic destinationsequenceddistance-vector routing (DSDV) for mobile computers, Proc. Conf. Commun. Archit., Protocols, (1994), pp.234-244.

[19] B. Radunovic, C. Gkantsidis, P. B. Key, and P. Rodriguez, Anoptimization framework for opportunistic multipath routing inwireless mesh networks, Proc. IEEE 27th Conf. Comput. Commun., (2008), pp.241-245.

[20] H. Rahul, H. Hassanieh, and D. Katabi, Sourcesync: A distributedwireless architecture for exploiting sender diversity, Proc. SIGCOMM, (2010), pp.171-182. 
Dynamic Path construction in Multi-Hop Wireless Networks

(This page is empty intentionally) 